Pacific

Journal of

Mathematics

AN INVERSE PROBLEM FROM SUB-RIEMANNIAN GEOMETRY

Thomas A. IVey 


\title{
AN INVERSE PROBLEM FROM SUB-RIEMANNIAN GEOMETRY
}

\author{
Thomas A. Ivey
}

\begin{abstract}
The geodesics for a sub-Riemannian metric on a threedimensional contact manifold $M$ form a 1-parameter family of curves along each contact direction. However, a collection of such contact curves on $M$, locally equivalent to the solutions of a fourth-order ODE, are the geodesics of a sub-Riemannian metric only if a sequence of invariants vanish. The first of these, which was first identified by Fels, determines if the differential equation is variational. The next two determine if there is a well-defined metric on $M$ and if the given paths are its geodesics.
\end{abstract}

\section{Introduction.}

In this note we discuss the problem of recovering the geometric structure of a three-dimensional contact manifold with a sub-Riemannian metric from the geodesics for the metric. (Sub-Riemannian metrics are also known as Carnot-Carathéodory metrics.) Since all the results herein will be local in nature, the manifold may be taken to be an open set $U \in \mathbb{R}^{3}$ with contact form $d y-z d x$, and we may assume that on contact planes the metric has the form

$$
g=E d x^{2}+F d x d z+G d z^{2},
$$

where $E, F, G$ are smooth functions on $U$ such that $g$ is positive definite. The geodesics form a collection of paths tangent to the contact structure, such that there is a 1-parameter family of distinct paths tangent to each contact direction at each point. Thus, part of the problem will be to determine which such collections of paths come from a sub-Riemannian metric.

As explained below, the paths are locally equivalent to the integral curves of a scalar fourth-order ODE. The variational multiplier problem for fourthorder ODE - i.e., the problem of characterizing equations which are, up to multiple, the Euler-Lagrange equations for a second-order Lagrangian - was solved by M. Fels [4]. Since sub-Riemannian geodesics arise as solutions of a variational problem, the present work is an extension of that of Fels; to avoid confusion, the notation of [4] will be used whenever possible. 


\section{Contact path geometries.}

In this section we review the construction of sub-Riemannian geodesics, and define a $G$-structure canonically associated to the geodesics as paths.

Let $M$ be an oriented three-manifold with contact distribution $\mathcal{D}$ and sub-Riemannian metric $g$. It is standard that one can associate to $g$ a $S O(2)$-structure $N$ inside the oriented coframe bundle $F(M)$, such that, for any coframing which is a local section of $N$, the forms $\left(\omega^{1}, \omega^{2}, \omega^{3}\right)$ of the coframing satisfy:

(i) $\omega^{3}$ annihilates the contact planes;

(ii) $\left(\omega^{1}\right)^{2}+\left(\omega^{2}\right)^{2}$ coincides with the metric on the contact planes; and, (iii) $\omega^{1} \wedge \omega^{2}$ gives the induced orientation.

Furthermore, we may specify $N$ uniquely by requiring that $d \omega^{3}=\omega^{1} \wedge$ $\omega^{2}$. Then $N$ has a connection form $\phi$ satisfying the following structure equations: ${ }^{1}$

$$
\begin{aligned}
d \omega^{1} & =\phi \wedge \omega^{2}+\left(a_{1} \omega^{1}+a_{2} \omega^{2}\right) \wedge \omega^{3} \\
d \omega^{2} & =-\phi \wedge \omega^{1}+\left(a_{2} \omega^{1}-a_{1} \omega^{2}\right) \wedge \omega^{3} \\
d \omega^{3} & =\omega^{1} \wedge \omega^{2} \\
d \phi & =K \omega^{1} \wedge \omega^{2} \bmod \omega^{3} .
\end{aligned}
$$

The functions $a_{1}, a_{2}$ are components of the torsion of $g$ and $K$ is the curvature. $^{2}$

Every oriented contact curve in $M$ has a lift to $N$ on which the forms $\omega^{2}$ and $\omega^{3}$ vanish. Applying the Griffiths formalism [7] to find the integral curves in $N$ of the Pfaffian system $\left\{\omega^{2}, \omega^{3}\right\}$ which are extremal curves for arclength $\int \omega^{1}$, we obtain the following characterization of sub-Riemannian geodesics: ${ }^{3}$

Proposition 1.1. Let $Z$ be the rank one affine subbundle of $T^{*} N$ on which the canonical one-form is $\sigma=\omega^{1}-x \omega^{3}, x \in \mathbb{R}$. (Forms on $Z$ are pulled back via $\pi: Z \rightarrow N$.) Then smooth geodesics are in 1-to-1 correspondence, via the submersion $Z \rightarrow M$, with integral curves of the Pfaffian system

\footnotetext{
${ }^{1}$ This result appears in [6], where it is attributed independently to Bryant-Hsu and to G. Wilkens. A detailed derivation can be found in [9].

${ }^{2}$ Clearly, taking the $\omega^{i}$ as an orthonormal coframe canonically associates to $g$ with Riemannian metric $\widehat{g}$ on $M$, which induces $g$ on $\mathcal{D}$, and defines a canonical foliation perpendicular to $\mathcal{D}$. The torsion tensor is the Lie derivative of $\widehat{g}$ along the leaves; if this vanishes, $g$ descends to any (locally defined) quotient surface by foliation, and $K$ is the Gauss curvature of the metric on that surface.

${ }^{3}$ See $[\mathbf{9}]$ and $[\mathbf{1 0}]$ for derivations of the geodesics by this and other methods.
} 
$\mathcal{F}=\left\{\theta_{0}, \theta_{1}, \theta_{2}, \theta_{3}\right\}$ on $Z$, where

$$
\begin{aligned}
& \theta_{0}=\omega^{3} \\
& \theta_{1}=\omega^{2} \\
& \theta_{2}=\phi-x \omega^{1} \\
& \theta_{3}=d x-a_{1} \omega^{1}-a_{2} \omega^{2} .
\end{aligned}
$$

Remark. For variational problems with differential constraints, it is in general not known under what conditions all extremal curves arise as projections of integral curves of the differential system formulated by Griffiths. For example, for sub-Riemannian metrics on a generic two-plane distribution in dimension four, exceptional extremal curves exist which do not come from the Griffiths system. Essentially, this is because these abnormal minimizers $[\mathbf{2}, \mathbf{1 0}]$ have few or no compactly supported variations that are tangent to the given distribution. However, by applying the regularity test given by $\mathrm{Hsu}[8]$, one can show that for a sub-Riemannian metric on a contact manifold, all geodesics arise via the Griffiths formalism. (Intuitively, enough variations exist because contact curves can be locally expressed in terms of an arbitrary function and its derivatives.)

Returning to the system $\mathcal{F}$ given above, let $L$ be the line field on $Z$ which is annihilated by $\mathcal{F}$. Integral curves of this line field push down via $\pi$ to give a 1-parameter family of curves through each point of $N$, and push down to $M$ to give a 1-parameter family of geodesics tangent to each contact direction.

Recall that the prolongation [2] of a contact manifold $M$ is the sub-bundle of the projectivization of $T M$ whose fibre consists of all contact directions at the basepoint in $M$. For a distribution $\mathcal{D}$ of two-planes on a three-manifold, the prolongation is a $\mathbb{P}^{1}$-bundle $\mathbb{P} \mathcal{D}$ over $M$. This bundle carries a canonical smooth two-plane distribution $\mathcal{D}^{\prime}$, defined as follows: For a nonzero vector $v \in D$, we say that a vector $w \in T_{[v]} \mathbb{P} \mathcal{D}$ is tangent to $\mathcal{D}^{\prime}$ if and only if $\pi_{*} w$ is a multiple of $v$, where $\pi: \mathbb{P D} \rightarrow M$ is the fibration. It follows that $\mathcal{D}^{\prime}$ is tangent to the fibres of $\pi$.

For example, let $\mathcal{D}$ be the contact distribution on sub-Riemannian threemanifold $M$. Then the canonical $S O(2)$-structure $N$ is a double cover of $\mathbb{P} \mathcal{D}$, by the map that sends coframe $\left(\omega^{1}, \omega^{2}, \omega^{3}\right) \in T_{x}^{*} M$ to the line in $T_{x} M$ annihilated by $\omega^{2}$ and $\omega^{3}$. It follows that $\mathcal{D}^{\prime}$ lifts to $N$ to be the two-plane field annihilated by $\omega^{2}$ and $\omega^{3}$.

The construction of the prolongation may be repeated, resulting each time in a $\mathbb{P}^{1}$-bundle over the previous space, carrying a canonical two-plane distribution. For example, $Z$ can be embedded as an open subset of the prolongation of the two-plane field on $N$ (the subset consisting of directions $v$ such that $\omega^{1}(v) \neq 0$ ) by sending a point in the fibre $Z_{u}$ to the line in $T_{u} N$ 
annihilated by $\theta_{0}, \theta_{1}$ and $\theta_{2}$. Under this embedding, the integral curves of the Pfaffian system $\mathcal{F}$ defined above become tangent to the contact planes on the prolongation of $N$. Moreover, under the natural lifting of the double cover $N \rightarrow \mathbb{P D}$ to a double cover from $Z$ to an open subset $U \subset \mathbb{P} \mathcal{D}^{\prime}$, these integral curves descend to give a well-defined foliation of $U$.

We will now generalize this situation by throwing away the metric.

Definition 1.2. Let $M^{3}$ be a contact manifold and let $P^{5}$ be the second prolongation of $M$. Let $L$ be a line field on $P$ which is tangent to the canonical two-plane distribution $\mathcal{D}^{\prime \prime}$ and everywhere transverse to the fibres of $\rho: P \rightarrow M$. Let $\mathcal{I}$ be the Pfaffian system on $P$ which annihilates $L$ and the fibres of $\rho$, and let $\mathcal{J}$ be the intersection of the retracting space $[\mathbf{1}]$ of $\mathcal{I}$ with the annihilator of $L$. Then $(P, L, \rho)$ defines a contact path geometry on $M$ if:

(i) The first derived system $\mathcal{I}^{\prime}$ is one-dimensional at each point of $P$;

(ii) $\mathcal{J}^{\prime}=\mathcal{I}$ at each point of $P$.

These two conditions need explaining. Because $L$ is transverse to the fibres, $\mathcal{I}$ is two-dimensional. If $\mathcal{I}$ were integrable (i.e., $\mathcal{I}^{\prime}=\mathcal{I}$, instead of being one-dimensional) then all paths through a given fibre $\rho^{-1}(x)$ would project down to a single contact curve on $M$, so that there would be only one path through $x \in M$. Condition (i) implies that $\mathcal{J}$ is three-dimensional at each point; it is automatic that $\mathcal{I} \subset \mathcal{J}^{\prime}$. If $\mathcal{J}$ were integrable, then integral surfaces of $\mathcal{J}$ would intersect $\rho^{-1}(x)$ in a 1-parameter family of curves; since each such surface would project down to a single contact curve in $M$, this would imply that there was only a 1-parameter family of paths through $x$.

Since $\mathcal{I}^{\prime}$ contains the pullback of a contact form on $M$, condition (i) also implies that $\mathcal{I}^{\prime \prime}=0$. Thus, the three-dimensional distribution containing $L$ and the kernel of $\rho_{*}$ is bracket-generating. That in turn guarantees, by Chow's theorem [3], that two arbitrary points in $M$ can be connected by a piecewise smooth sequence of paths.

In practice, we will work locally, assuming that $L$ is defined on an open subset $U \subset P$. Now the question of which path geometries are sub-Riemannian becomes that of which such line fields $L$ are locally diffeomorphic to the line field on $Z$ associated to some sub-Riemannian metric on $M$, under diffeomorphisms which respect the fibrations and two-plane distributions.

Proposition 1.3. Given a contact path geometry we can construct, in a neighbourhood of any point $q \in P$, a coframe $\left(\sigma, \theta_{0}, \theta_{1}, \theta_{2}, \theta_{3}\right)$ such that:

1. $v \in T P$ projects down to be a contact direction on $M$ if and only if $\theta_{0}(v)=0$

2. $\mathcal{I}=\left\{\theta_{0}, \theta_{1}\right\}$

3. $\mathcal{J}=\left\{\theta_{0}, \theta_{1}, \theta_{2}\right\}$

4. $L^{\perp}=\left\{\theta_{0}, \theta_{1}, \theta_{2}, \theta_{3}\right\}$. 
Moreover, these forms satisfy

$$
d \theta_{i} \equiv \theta_{i+1} \wedge \sigma \bmod \theta_{0}, \ldots, \theta_{i}, \quad 0 \leq i \leq 2 .
$$

These will be called 0-adapted coframes for the contact path geometry.

Proof. Let $\rho(q)=x \in M$. On a neighbourhood $V$ of $x$, there exists a contact form $\theta_{0}$, and 1-forms $\sigma, \theta_{1}$ such that $d \theta_{0} \equiv \theta_{1} \wedge \sigma \bmod \theta_{0}$. Pull these forms back to $U=\rho^{-1}(V) \subset P$; we will shrink $U$ when necessary. Since $\sigma, \theta_{1}$ both restrict to be zero along the fibres of $\rho$, they cannot be independent modulo $\mathcal{I}$. Therefore we can arrange, by adding multiples of $\sigma$, that $\theta_{1} \in \mathcal{I}$. (Note that now $\theta_{1}$ is no longer the pullback of a form on $V$.) Since $\theta_{0}, \theta_{1} \in L^{\perp}$, then $\sigma \notin L^{\perp}$. Since $\theta_{0} \in \mathcal{I}^{\prime}$, then $d \theta_{1} \neq 0 \bmod \mathcal{I}$.

Since $\theta_{0}, \theta_{1}, \sigma$ span an integrable system, then there will be a smooth 1form $\theta_{2}$ on $U$ that $d \theta_{1} \equiv \theta_{2} \wedge \sigma \bmod \mathcal{I}$. (Since $\mathcal{I}^{\prime}$ is one-dimensional at each point, $\theta_{2}$ is nonzero on $U$.) By adding multiples of $\sigma$, we can arrange that $\theta_{2} \in L^{\perp}$, giving condition 3 . Because $\mathcal{J}^{\prime} \neq \mathcal{J}$, there must be a nonzero 1-form $\theta_{3}$ on $U$ such that $d \theta_{2} \equiv \theta_{3} \wedge \sigma \bmod \mathcal{J}$. We can similarly arrange that $\theta_{3} \in L^{\perp}$.

Remark. The above proposition could also be proved just using the assumption that $L$ is a line field on a five-manifold $P$, carrying a two-dimensional Pfaffian system $\mathcal{I}$ annihilating $L$, such that $\mathcal{I}, \mathcal{J}$ satisfy conditions (i, ii) in Defn. 1.2. The contact structure and the submersion to $M$ can be recovered from $\mathcal{I}^{\prime}$ and the retracting space $\mathcal{C}\left(\mathcal{I}^{\prime}\right)$ respectively.

Corollary 1.4. In some neighbourhood $U$ of any given point $q \in P$, there exist coordinates $x, y_{0}, y_{1}, y_{2}, y_{3}$ such that, for some function $F$ on $U$,

$$
\begin{aligned}
\sigma & =-d x \\
\theta_{0} & =d y_{0}-y_{1} d x \\
\theta_{1} & =d y_{1}-y_{2} d x \\
\theta_{2} & =d y_{2}-y_{3} d x \\
\theta_{3} & =d y_{3}-F\left(x, y_{0}, y_{1}, y_{2}, y_{3}\right) d x
\end{aligned}
$$

is a 0-adapted coframe. Consequently, paths in $P$ are locally equivalent to the solutions of the fourth-order ODE

$$
y^{\prime \prime \prime \prime}=F\left(x, y, y^{\prime}, y^{\prime \prime}, y^{\prime \prime \prime}\right) .
$$

Proof. The structure equations (1) enable us to apply the Goursat normal form theorem [1] to system $\mathcal{J}$. This gives $\mathcal{J}=\left\{\theta_{0}, \theta_{1}, \theta_{2}\right\}$, in terms of the forms defined here. Since $\mathcal{I}=\left\{\theta_{0}, \theta_{1}\right\}$, then $d x \notin L^{\perp}$, and so there exists some function $F$ such that $d y_{3}-F\left(x, y_{0}, y_{1}, y_{2}, y_{3}\right) d x=0$ along the paths in $U$. 
The set of 0 -adapted coframes $\left(\sigma, \theta_{0}, \theta_{1}, \theta_{2}, \theta_{3}\right)$ for given contact path geometry forms a principal bundle $B_{0}$ over $P$, with ten-dimensional structure group $G_{0} \subset G L(5, \mathbb{R})$ consisting of matrices of the form

$$
\left(\begin{array}{ccccc}
a & * & * & 0 & 0 \\
0 & b & 0 & 0 & 0 \\
0 & * & a^{-1} b & 0 & 0 \\
0 & * & * & a^{-2} b & 0 \\
0 & * & * & * & a^{-3} b
\end{array}\right)
$$

(The stars indicate arbitrary entries.) This is precisely the $G$-structure that Fels associates to a fourth-order ODE up to contact transformation (cf. [4], Lemma 3.1). Since the path geometry can be recovered uniquely from the $G$-structure, we will treat the two notions as synonymous.

\section{Variational and sub-Riemannian path geometries.}

The goal of Cartan's method of equivalence [5] is, for a given $G$-structure, to find a sub-bundle, with reduced structure group, on which there exists a unique connection. Like the Levi-Civita connection in Riemannian geometry, this is typically obtained by fixing the value of all or part of the torsion of the connection. Then, invariants may be extracted from the remaining torsion or the curvature of the connection.

We begin with Fels' result for $G_{0}$-structures of coframes satisfying (1). This gives a reduction of structure to the subgroup $G_{1} \subset G_{0}$ consisting of matrices of the form

$$
\left(\begin{array}{ccccc}
a & 0 & 0 & 0 & 0 \\
0 & b & 0 & 0 & 0 \\
0 & 0 & a^{-1} b & 0 & 0 \\
0 & 0 & 0 & a^{-2} b & 0 \\
0 & 0 & 0 & 0 & a^{-3} b
\end{array}\right) \cdot \exp \left(\begin{array}{ccccc}
0 & 0 & 0 & 0 & 0 \\
0 & 0 & 0 & 0 & 0 \\
0 & c & 0 & 0 & 0 \\
0 & 0 & \frac{4}{3} c & 0 & 0 \\
0 & 0 & 0 & c & 0
\end{array}\right)
$$

In terms of path geometry, the result is:

Theorem 1 (Fels [4]). Let $B_{0} \searrow P$ define a contact path geometry. Then there is a sub-bundle $B_{1}$ with three-dimensional structure group $G_{1}$, on which there exists a unique equivariant connection satisfying the following structure equations:

$$
\begin{aligned}
d \sigma= & \alpha \wedge \sigma+\theta_{0} \wedge\left(T_{1} \theta_{1}+T_{2} \theta_{2}+T_{3} \theta_{3}\right)+\theta_{1} \wedge\left(T_{4} \theta_{2}+T_{5} \theta_{3}\right) \\
d \theta_{0}= & \beta \wedge \theta_{0}+\sigma \wedge \theta_{1} \\
d \theta_{1}= & (\beta-\alpha) \wedge \theta_{1}+\gamma \wedge \theta_{0}+\sigma \wedge \theta_{2} \\
d \theta_{2}= & (\beta-2 \alpha) \wedge \theta_{2}+\frac{4}{3} \gamma \wedge \theta_{1}+\sigma \wedge \theta_{3} \\
d \theta_{3}= & (\beta-3 \alpha) \wedge \theta_{3}+\gamma \wedge \theta_{2}+\sigma \wedge\left(I_{0} \theta_{0}+I_{1} \theta_{1}\right) \\
& +T_{6} \theta_{0} \wedge \theta_{1}+T_{7} \theta_{0} \wedge \theta_{2}+T_{8} \theta_{1} \wedge \theta_{2} .
\end{aligned}
$$


[The one-forms $\alpha, \beta, \gamma$ are connection forms, and $I_{0}, I_{1}, T_{1}, \ldots T_{8}$ are components of the torsion of the connection.]

Moreover, assuming $P$ is locally defined by a fourth-order ODE (3), solutions of that ODE are critical curves for a second-order Lagrangian if and only if the relative invariants $I_{1}$ and $T_{5}$ both vanish identically on $B_{1}$. In that case, $T_{8}$ also vanishes.

The essence of Fels' proof of the second statement is exhibiting a two-form on $B_{1}$,

$$
\omega=m\left(\theta_{0} \wedge \theta_{3}-\theta_{1} \wedge \theta_{2}\right),
$$

where $m$ is a nonzero function, such that $\omega$ is closed and $G_{1}$-invariant. (In fact, $d \log m=3 \alpha-2 \beta$, and, as Fels notes, the structure equations imply that that one-form is closed in the variational case.) It then follows that $\omega$ is the exterior derivative of the Poincaré-Cartan form associated to a Lagrangian on the space of 2-jets.

We will speak of a path geometry for which $I_{1}, T_{5}, T_{8}$ vanish identically as being variational.

Example 1. Consider the second-order Lagrangian $\int e^{-3 y^{\prime \prime}} d x$, for which the Euler-Lagrangian equations are, up to multiple,

$$
y^{\prime \prime \prime \prime}-3\left(y^{\prime \prime \prime}\right)^{2}=0 \text {. }
$$

The coframe (2) gives a section of the bundle $B_{0}$ defining the corresponding $G_{0}$-structure on $J^{3}(\mathbb{R}, \mathbb{R})$. This coframe may be modified to give the following section of the reduced structure $B_{1}$ :

$$
\begin{aligned}
\theta_{0} & =d y_{0}-y_{1} d x \\
\theta_{1} & =d y_{1}-y_{2} d x \\
\theta_{2} & =d y_{2}-y_{3} d x-y_{3} \theta_{1}+\frac{3}{10} y_{3}^{2} \theta_{0} \\
\theta_{3} & =d y_{3}-3 y_{3} d y_{2}-\frac{3}{10} y_{3}^{2} \theta_{1}+\frac{6}{5} y_{3}^{3} \theta_{0} \\
\sigma & =d x+\theta_{1}-\frac{3}{5} y_{3} \theta_{0} .
\end{aligned}
$$

Of course, the torsion satisfies $I_{1}=T_{5}=T_{8}=0$, but one may also compute ${ }^{4}$ that $T_{2}=\frac{12}{5} y_{3}, T_{3}=\frac{3}{5}$ and $T_{4}=-1$ along this section of $B_{1}$.

\footnotetext{
${ }^{4}$ In order to evaluate the torsion components along a given section of $B_{1}$, one must determine the values of the connection forms in terms of the given coframe. To do this, begin with the $d \theta_{0}$ equation (4b), which determines $\beta$ modulo $\theta_{0}$. One may set $\beta=\beta_{0}+b \theta_{0}$, where $\beta_{0}$ is any form satisfying (4b) and $b$ is not yet determined. Then (4c) determines $\alpha$ and $\gamma$ modulo $\theta_{0}, \theta_{1}$. In fact, one may set

$$
\begin{aligned}
& \alpha=\alpha_{0}+a \theta_{0}+z \theta_{1} \\
& \gamma=\gamma_{0}-a \theta_{1}+c \theta_{0} .
\end{aligned}
$$
}

Now (4a) determines $z$ while $(4 \mathrm{~d}),(4 \mathrm{e})$ determine $a, b$ and $c$. 
Example 2 (sub-Riemannian geometry). Let $Z$ be the five-manifold of Proposition 1.1. It is easy to verify that the 1 -forms given there, when rounded out by $\sigma=\omega^{1}-x \omega^{3}$, form a 0 -adapted coframe for the corresponding contact path geometry. We may adapt the coframe to obtain a section of the reduced bundle $B_{1} \searrow Z$ :

$$
\begin{aligned}
\theta_{0} & =\omega^{3} \\
\theta_{1} & =\omega^{2} \\
\theta_{2} & =\phi-x \omega^{1}+A \omega^{3} \\
\theta_{3} & =d x-a_{1} \omega^{1}-\left(a_{2}+A\right) \omega^{2}+B \omega^{3} \\
\sigma & =\omega^{1}-\frac{3}{5} x \omega^{3}
\end{aligned}
$$

with

$$
\begin{aligned}
A & =\frac{1}{10}\left(a_{2}+3 x^{2}-3 K\right) \\
B & =\frac{1}{10}\left(s_{2}-3 k_{1}-6 a_{1} x-21 b_{1}\right),
\end{aligned}
$$

where $K$ is the scalar curvature, and the $b_{i}, s_{i}$ and $k_{i}$ are defined on $N$ by

$$
\left.\begin{array}{rl}
d a_{1} & \equiv 2 a_{2} \phi+\left(s_{1}+b_{2}\right) \omega^{1}+\left(s_{2}+b_{1}\right) \omega^{2} \\
d a_{2} & \equiv-2 a_{1} \phi+\left(s_{2}-b_{1}\right) \omega^{1}+\left(b_{2}-s_{1}\right) \omega^{2} \\
d K & \equiv k_{1} \omega^{1}+k_{2} \omega^{2}
\end{array}\right\} \bmod \omega^{3} .
$$

Again, one may compute that $I_{1}=T_{5}=T_{8}=0$, confirming that the path geometry is variational, while $T_{2}=0, T_{3}=\frac{3}{5}$, and $T_{4}=-1$ for this coframe.

The fact that we obtained the same values for $T_{3}$ and $T_{4}$ as those from a general second-order Lagrangian hints at further relations among the torsion components. One uncovers one of these by deriving the refined structure equations:

Proposition 2.1. Let $B_{1}$ be the canonical $G_{1}$-structure for a variational path geometry. Then there exist functions $U_{1}, U_{2}$ on $B_{1}$ such that the connection forms satisfy

$$
\begin{aligned}
& d \alpha=\frac{2}{3} d \beta \\
& d \beta=\sigma \wedge \gamma-\tau \wedge \theta_{1}-3 \nu \wedge \theta_{0} \\
& d \gamma \equiv \gamma \wedge \alpha-\tau \wedge \theta_{2}-\nu \wedge \theta_{1} \bmod \theta_{0}
\end{aligned}
$$

where

$$
\begin{aligned}
& \tau=T_{1} \theta_{1}+T_{2} \theta_{2}+T_{3} \theta_{3} \\
& \nu=U_{1} \theta_{1}+U_{2} \theta_{2}-T_{2} \theta_{3}+T_{7} \sigma .
\end{aligned}
$$


The torsion components satisfy $T_{3}=-\frac{3}{5} T_{4}$ and

$$
\begin{array}{ll}
d T_{1} \equiv T_{1}(2 \alpha-2 \beta)-\frac{4}{3} T_{2} \gamma-2 U_{1} \sigma & \bmod \theta_{0}, \theta_{1}, \theta_{2}, \theta_{3} \\
d T_{2} \equiv T_{2}(3 \alpha-2 \beta)-\frac{2}{5} T_{4} \gamma-\left(T_{1}+2 U_{2}\right) \sigma & \bmod \theta_{0}, \theta_{1}, \theta_{2}, \theta_{3} \\
d T_{4} \equiv T_{4}(4 \alpha-2 \beta)-\frac{5}{3} T_{2} \sigma & \bmod \theta_{0}, \theta_{1}, \theta_{2} .
\end{array}
$$

The above equations indicate that $T_{4}$ is a relative invariant on $B_{1}$, i.e., it varies along the fibres only by scaling. Moreover, they indicate that the quadratic form $g=\sigma^{2}-T_{4} \theta_{1}^{2}$ is well-defined, up to multiple and modulo $\theta_{0}$, on $N$. For, suppose $v$ is a vector field on $B_{1}$ which is annihilated by $\sigma, \theta_{0}, \theta_{1}, \theta_{2}$. Then computing the Lie derivative of $g$ gives

$$
\begin{aligned}
\mathcal{L}_{v}(g) & \left.\left.=2 \sigma \circ(v\lrcorner d \sigma)-2 T_{4} \theta_{1} \circ(v\lrcorner d \theta_{1}\right)-(v\lrcorner d T_{4}\right) \theta_{1}^{2} \\
& \equiv 2(v\lrcorner \alpha)\left[\sigma^{2}-T_{4} \theta_{1}^{2}\right] \bmod \theta_{0} .
\end{aligned}
$$

Example 2 shows that, if the contact path geometry comes from a subRiemannian metric, then this quadratic form must coincide, up to multiple, with the metric. Matters being so, we will say that a variational geometry is nondegenerate if $T_{4} \neq 0$ everywhere, ${ }^{5}$ and definite if $T_{4}$ is negative everywhere. Assuming the latter is the case, then we may normalize $T_{2}$ and $T_{4}$ to have the same values as in Example 2.

Proposition 2.2. Let $B_{1}$ be the canonical $G_{1}$-structure for a definite variational path geometry. Then there is a sub-bundle $B_{2} \subset B_{1}$ on which

$$
T_{2}=0 \text { and } T_{4}=-1 .
$$

On $B_{2}$ there exist smooth functions $W_{0}, W_{1}, W_{2}, G_{0}, G_{1}, G_{2}, G_{3}$, and $H$ such that

$$
\begin{aligned}
& \beta=2 \alpha+W_{0} \theta_{0}+W_{1} \theta_{1}+W_{2} \theta_{2} \\
& \gamma=H \sigma-3\left(G_{0} \theta_{0}+G_{1} \theta_{1}+G_{2} \theta_{2}+G_{3} \theta_{3}\right) .
\end{aligned}
$$

Proof. Structure equations (9) and (10) show that we may first pass to the sub-bundle where $T_{4}=-1$ and then move along the fibres in a direction dual to $\gamma$ to pass to the sub-bundle where $T_{2}=0$. Once there, these equations show that $\beta-2 \alpha$ and $\gamma$ restrict to have the above form. Of course, (9) shows that $\frac{2}{5} H=T_{1}+2 U_{2}$.

\footnotetext{
${ }^{5}$ Suppose a variational path structure has $T_{3}$ and $T_{4}$ identically zero; the refined structure equations show that $T_{2}=0$ also. Recall that the system which restricts to be zero along the fibres of $\rho: P \rightarrow M^{3}$ is spanned by $\sigma, \theta_{0}, \theta_{1}$. Since $d \sigma \equiv T_{1} \theta_{0} \wedge \theta_{1} \bmod \sigma$, vectors that are in the kernel of $\sigma$ push down to give a well-defined plane field on $M$. These planes intersect the contact planes in a distinguished family of contact directions, which are null lines with respect to $g$.
} 
On $B_{2}$, the structure equations (4) take the form

$$
\begin{aligned}
d \sigma & =\alpha \wedge \sigma+\theta_{0} \wedge\left(T_{1} \theta_{1}+\frac{3}{5} \theta_{3}\right)-\theta_{1} \wedge \theta_{2} \\
d \theta_{0} & =2 \alpha \wedge \theta_{0}+\sigma \wedge \theta_{1} \\
d \theta_{1} & =(\beta-\alpha) \wedge \theta_{1}+\gamma \wedge \theta_{0}+\sigma \wedge \theta_{2} \\
d \theta_{2} & =(\beta-2 \alpha) \wedge \theta_{2}+\frac{4}{3} \gamma \wedge \theta_{1}+\sigma \wedge \theta_{3} \\
d \theta_{3} & =(\beta-3 \alpha) \wedge \theta_{3}+\gamma \wedge \theta_{2}+I_{0} \sigma \wedge \theta_{0}+T_{6} \theta_{0} \wedge \theta_{1}+T_{7} \theta_{0} \wedge \theta_{2}
\end{aligned}
$$

with $\beta$ given by (11).

It's clear that the fibres of $B_{2}$ are one-dimensional (with $\alpha$ as the only independent connection form), and the structure group of $B_{2}$ is simply $\mathbb{R}^{*}$. A element $\lambda \neq 0$ of this group acts on sections of $B_{2}$ by

$$
g_{\lambda} \cdot\left(\sigma, \theta_{0}, \theta_{1}, \theta_{2}, \theta_{3}\right)=\left(\lambda \sigma, \lambda^{2} \theta_{0}, \lambda \theta_{1}, \theta_{2}, \lambda^{-1} \theta_{3}\right) .
$$

Structure equations (7) show that $g_{\lambda}^{*} \alpha=\alpha, g_{\lambda}^{*} \beta=\beta$, and $g_{\lambda}^{*}(\gamma)=\lambda^{-1} \gamma$. Then the action on the new torsion is clearly

$$
\begin{aligned}
g_{\lambda} \cdot\left(W_{0}, W_{1}, W_{2}\right) & =\left(\lambda^{-2} W_{0}, \lambda^{-1} W_{1}, W_{2}\right), \\
g_{\lambda} \cdot\left(H, G_{0}, G_{1}, G_{2}, G_{3}\right) & =\left(\lambda^{-2} H, \lambda^{-3} G_{0}, \lambda^{-2} G_{1}, \lambda^{-1} G_{2}, G_{3}\right) .
\end{aligned}
$$

In particular, $W_{2}, G_{3}$, and the ratios $G_{1}: W_{0}$ and $G_{2}: W_{1}$ are invariant under the scaling action.

We should expect this scaling to be present, since two sub-Riemannian metrics which differ by a constant factor have the same geodesics and hence define the same path geometry. For purposes of constructing a specific metric, we will need to choose a section of $B_{2}$. Since $3 \alpha-2 \beta$ is closed, integrals of this one-form comprise a canonical codimension-one foliation of $B_{2}$ which is transverse to fibres and invariant under the scaling action.

Definition 2.3. A section of $B_{2}$ along which

$$
3 \alpha-2 \beta=0
$$

will be called a canonical section of $B_{2}$, or a canonical coframe on $P$. It follows from (11) that

$$
\alpha=-2\left(W_{0} \theta_{0}+W_{1} \theta_{1}+W_{2} \theta_{2}\right)
$$

along a canonical section.

One can check that the coframing constructed in Example 2 is a canonical coframe. Since such coframings are unique up to scale, it follows that if a path geometry comes from a sub-Riemannian metric, then in terms of a canonical coframe that metric must be $g=\sigma^{2}+\left(\theta_{1}\right)^{2}$.

Proposition 2.4. Let $P$ be a definite variational path geometry for contact manifold $M^{3}$ and $\left(\sigma, \theta_{0}, \theta_{1}, \theta_{2}, \theta_{3}\right)$ a canonical coframe on $P$. Then $g=$ $\sigma^{2}+\left(\theta_{1}\right)^{2}$ gives a well-defined metric on the contact planes of $M$ if and only if $W_{2}$ is identically zero on $P$. 
Proof. Let $v$ be any vector field on $P$ tangent to the fibres of the projection $\rho: P \rightarrow M$. Since $v$ is annihilated by $\theta_{0}, \theta_{1}$ and $\sigma$,

$$
\begin{aligned}
\mathcal{L}_{v}(g) & \left.\equiv(v\lrcorner \alpha)(\sigma)^{2}+(v\lrcorner(\beta-\alpha)\right)\left(\theta_{1}\right)^{2} \bmod \theta_{0} \\
& \equiv-W_{2}\left(v-\theta_{2}\right)\left(2(\sigma)^{2}+\left(\theta_{1}\right)^{2}\right) .
\end{aligned}
$$

Although the coframe (5) is not a section of $B_{2}$, it can be adjusted so that $T_{2}=0$, whereupon we see that $W_{2}$ is nonzero for Example 1 .

For the rest of this section we will assume that $W_{2}$ is identically zero. It remains to be seen if the given paths on $M^{3}$-which are projections of the integral curves of the line field $L$ - are the geodesics of the sub-Riemannian metric we have constructed. To investigate this further, we will need the torsion identities

$$
G_{3}=0, \quad G_{2}=W_{1},
$$

which result from computing $d\left(d \theta_{1}\right)=0$ using the structure equations (13) and equations (12),(14) and (15) with $W_{2}=0$.

Remark. One might wonder if other identities hold among the remaining torsion coefficients $G_{0}, G_{1}, H, I_{0}, T_{1}, T_{6}, T_{7}, W_{0}, W_{1}$ as a result of our assumption that $W_{2}=0$. However, no further identities arise, and this is proved by showing that the exterior differential system defining a $G$ structure satisfying the structure equations on $B_{2}$ with $W_{2}=0$ is involutive.

Theorem 2. Let $P$ be a definite variational path geometry with $W_{2}$ identically zero, and let $\left(\sigma, \theta_{0}, \theta_{1}, \theta_{2}, \theta_{3}\right)$ be a fixed canonical coframe on $P$. Then the paths in $P$ project to be geodesics in $M$ for the sub-Riemannian metric of Proposition 2.4 if and only if $G_{1}=2 W_{0}$ identically on $P$.

Proof. Let $N$ be the quotient of $P$ by the foliation by integral curves of the system $\mathcal{I}^{(1)}=\left\{\sigma, \theta_{0}, \theta_{1}, \theta_{2}\right\}$. Each contact curve in $M$ has a unique lift to $N$ as an integral curve of $\mathcal{I}=\left\{\theta_{0}, \theta_{1}\right\}$. Clearly, arclength is measured along these lifts by the integral of $\sigma$ modulo $\mathcal{I}$. However, the form $\sigma$ on $P$ does not descend to be well-defined on $N$, as shown by

$$
\begin{aligned}
d \sigma & =\alpha \wedge \sigma+\theta_{0} \wedge\left(T_{1} \theta_{1}+T_{3} \theta_{3}\right)+T_{4} \theta_{1} \wedge \theta_{2} \\
& \equiv \frac{3}{5} \theta_{0} \wedge \theta_{3} \bmod \Lambda^{2} \mathcal{I}^{(1)} .
\end{aligned}
$$

Computing $d^{2} \theta_{0}=0$ shows that

$$
d W_{1} \equiv\left(G_{1}-W_{0}\right) \sigma+\frac{1}{5} \theta_{3} \bmod \mathcal{I},
$$

and this, together with $d \theta_{0} \equiv 0 \bmod \Lambda^{2} \mathcal{I}^{(1)}$, shows that the 1 -form

$$
\widetilde{\sigma}=\sigma+3 W_{1} \theta_{0}
$$

is well-defined on $N$. 
Now arclength with respect to the metric may be measured on the integral curves of $\mathcal{I}$ by the Lagrangian $\int \widetilde{\sigma}$. We will apply the Griffiths formalism [7] to investigate which of these are geodesics for $g$. Then, we will try to find conditions under which these curves coincide with the projections of the paths in $P$ under $\pi: P \rightarrow N$.

Let $\xi=\widetilde{\sigma}+x \theta_{0}+y \theta_{1}$ on $Y=N \times \mathbb{R}^{2}$. Then one finds that the two-form $d \xi$ is of full rank on $Y$, except where $y=0$. Accordingly, let $\xi=\widetilde{\sigma}+x \theta_{0}$ on $Z=N \times \mathbb{R}$. Now one computes that

$$
d \xi \equiv\left(d x+\left(3 G_{1}-W_{0}\right) \sigma\right) \wedge \theta_{0}+\left(\theta_{2}+\left(x+5 W_{1}\right) \sigma\right) \wedge \theta_{1} \bmod \theta_{0} \wedge \theta_{1} .
$$

Let $\mathcal{K}$ be the rank four Pfaffian system on $Z$ spanned by the four one-forms on the right in (17):

$$
\mathcal{K}=\left\{\theta_{0}, \theta_{1}, \theta_{2}+\left(x+5 W_{1}\right) \sigma, d x+\left(3 G_{1}-W_{0}\right) \sigma\right\} .
$$

According to the Griffiths formalism, integral curves of $\mathcal{K}$ project to be extremal curves for $\int \widetilde{\sigma}$ on $N$. These coincide with the projections of the paths in $P$ if and only if, in a neighbourhood $U$ of each point of $P$, there is a local diffeomorphism $\varphi: U \rightarrow Z$ such that $\varphi^{*} \mathcal{K}$ coincides with $L^{\perp}=$ $\left\{\theta_{0}, \theta_{1}, \theta_{2}, \theta_{3}\right\}$, the Pfaffian system on $P$ which defines the paths. (The diffeomorphism would follow from the identification of paths with geodesics on $N$.) The form $\varphi^{*}\left(\theta_{2}+\left(x+5 W_{1}\right) \sigma\right)$ belongs in $L^{\perp}$ if and only if $\varphi^{*} x=$ $-5 W_{1}$. Then, by $(16)$,

$$
\varphi^{*}\left(d x+\left(3 G_{1}-W_{0}\right) \sigma\right) \equiv\left(4 W_{0}-2 G_{1}\right) \sigma \bmod L^{\perp},
$$

showing that $\varphi^{*} \mathcal{K}=L^{\perp}$ if and only if $G_{1}=2 W_{0}$.

\section{More examples.}

The results of the previous section may be surprising. For, one could reason that, once a path geometry is known to be variational, it must arise from a second-order Lagrangian, of the form

$$
\int L\left(x, y, y^{\prime}, y^{\prime \prime}\right) d x
$$

satisfying the nondegeneracy condition $\partial^{2} L / \partial\left(y^{\prime \prime}\right)^{2} \neq 0$. Then $L$ is of the form

$$
L\left(x, y, y^{\prime}, y^{\prime \prime}\right)=\sqrt{E+F y^{\prime \prime}+G\left(y^{\prime \prime}\right)^{2}},
$$

for some functions $E, F, G$ of $x, y, y^{\prime}$, if and only if $L$, as a function of $y^{\prime \prime}$, satisfies a certain third-order ODE. In other words, it seems like only one extra condition must be satisfied in order for the Lagrangian to be length with respect to a sub-Riemannian metric. Instead, we find that two scalar conditions (in addition to the Fels variational condition) must hold in order for the metric to be well-defined and in order for its extremals to coincide 
with the given paths. (The reader should note that the above remark about involutivity implies that the condition $G_{1}=2 W_{0}$ is independent from $W_{2}=$ 0 .) It would be interesting to find examples of variational path geometries (equivalently, variational fourth-order ODE) which are nondegenerate, and for which $W_{2}=0$, but the extremals of the associated metric do not coincide with the given paths.

Fels [4] calculates values for relative invariants $I_{1}$ and $T_{5}$ for a general fourth-order ODE (3):

$$
\begin{gathered}
T_{5}=\frac{1}{6} \frac{\partial^{3} F}{\partial y_{3}^{3}} \\
I_{1}=\frac{\partial F}{\partial y_{1}}+\frac{1}{2} \frac{\partial F}{\partial y_{2}} \frac{\partial F}{\partial y_{3}}+\frac{1}{8}\left(\frac{\partial F}{\partial y_{3}}\right)^{3}-\frac{d}{d x}\left(\frac{\partial F}{\partial y_{2}}+\frac{3}{8}\left(\frac{\partial F}{\partial y_{3}}\right)^{2}\right)+\frac{1}{2} \frac{d^{2}}{d x^{2}} \frac{\partial F}{\partial y_{3}} .
\end{gathered}
$$

(Here, we use the shorthand $y_{k}$ for $d^{k} y / d x^{k}$, and the total derivative $d / d x$ is computed using the ODE.) So, the right-hand side of a variational fourthorder ODE must be at most quadratic in $y_{3}$. In particular, all linear fourthorder ODEs are variational. However, $T_{4}=0$ for all of these, and so they are degenerate in the sense defined earlier.

Consider the class of nonlinear equations defined by

$$
y^{\prime \prime \prime \prime}=f\left(x, y, y_{1}, y_{2}\right) y_{3}^{2} .
$$

The Fels conditions $I_{1}=T_{5}=0$ imply that such an equation is variational if and only if $f$ does not depend on $y$, and is of the form

$$
f\left(x, y_{1}, y_{2}\right)=g_{0}(q, r)+x g_{1}(q, r), \quad q=y_{2}, r=y_{1}-x y_{2}
$$

for functions $g_{0}$ and $g_{1}$ that satisfy

$$
\frac{\partial g_{1}}{\partial q}+\frac{\partial g_{0}}{\partial r}=0
$$

Using the same adapted coframe as Fels, we calculate that

$$
T_{4}=-\frac{1}{9}\left(f^{2}+3 f_{q}\right) .
$$

To obtain nondegenerate examples, we will confine ourselves to functions $f$ for which $T_{4} \neq 0$. As in Proposition 2.2, we modify the coframe so as to get $T_{2}=0$ and $T_{4}=-1$. After computing the new connection forms, we find that $W_{2}=0$ if and only if

$$
9 f_{q q}+18 f f_{q}+4 f^{3}=0 .
$$

This equation, when taken together with the partial differential equations for $f$ implied by the $I_{1}=0$, leads to two other second-order equations for $f$ and three third-order equations, forming a Frobenius system whose solutions depend on five constants of integration.

However, for these examples the solutions of the variational fourth-order ODE (18) are never the geodesics of the canonical sub-Riemannian metric 
given by Proposition 2.4. For, with (21) and its consequences taken into account, we find that $W_{0}$ is independent of $y_{3}$, but $G_{1}$ is a cubic polynomial in $y_{3}$ with leading coefficient $\left(f^{2}+3 f_{q}\right)^{3 / 2}$. Thus, $G_{1}=2 W_{0}$ cannot hold, because of our nondegeneracy condition.

Acknowledgements. I am grateful to Ian Anderson and Mark Fels, and to the referee, for valuable comments and suggestions. Most of the calculations in this paper have been made using Maple $\mathrm{V}$ and Maple 6.

\section{References}

[1] R. Bryant, S.-S. Chern, R.B. Gardner, P.A. Griffiths and H. Goldschmidt, Exterior Differential Systems, Springer, 1991, MR 92h:58007, Zbl 0726.58002.

[2] R. Bryant and L. Hsu, Rigidity of integral curves of rank 2 distributions, Invent. Math., 114 (1993), 435-461, MR 94j:58003, Zbl 0807.58007.

[3] W.L. Chow, Über systeme von linearen partiellen differentialgleichungen erster ordnung, Math. Annalen, 117 (1939), 98-105, MR 1,313d, Zbl 0022.02304.

[4] M. Fels, The inverse problem of the calculus of variations for scalar fourth-order ordinary differential equations, Trans. Amer. Math. Soc., 348(12) (1996), 5007-5029, MR 97f:49047, Zbl 0879.34016.

[5] R.B. Gardner, The Method of Equivalence and Its Applications, SIAM, Philadelphia, 1989, MR 91j:58007, Zbl 0694.53027.

[6] Z. Ge, On sub-Riemannian metrics and $\widetilde{\mathrm{SL}}_{2}-$ geometry, C.R. Math. Rep. Acad. Sci. Canada, 15(4) (1993), 149-154, MR 94h:57020, Zbl 0805.53036.

[7] P.A. Griffiths, Exterior Differential Systems and the Calculus of Variations, Birkhäuser, 1983, MR 84h:58007, Zbl 0512.49003.

[8] L. Hsu, Calculus of variations via the Griffiths formalism, J. Diff. Geom., 36 (1992), 551-589, MR 94a:58003, Zbl 0768.49014.

[9] W.K. Hughen, The Sub-Riemannian Geometry of Three-Manifolds, Ph.D. Thesis, Duke University, 1995.

[10] R. Montgomery, A survey of singular curves in sub-Riemannian geometry, J. Dynam. Control Systems, 1(1) (1995), 49-90, MR 95m:53060, Zbl 0941.53021.

Received May 31, 2001 and revised October 16, 2001.

Dept. of Mathematics

College of Charleston

Charleston SC 29424

E-mail address: ivey@math.cofc.edu 